\title{
Energetic Surface Instruct for Faceted Result Finding Engines
}

\author{
Golamari Sowmya, D.Rammohanreddy
}

\begin{abstract}
Faceted reading is huge made use of in net electric powered poweredpowered shops and moreover subjects courting locations. In the ones situations, a corporation organized run-thru of additives is usually performed. This approach recollections 2 crucial issues. Initial, one want to feature a number one amount of your opportunity to devise a suitable study. Second, with a enterprise corporation corporation evaluate of additives it's going to in truth upward push up that a component ultimately ultimately subsequently ends up useless if all inventory that art work with the questions square motion regarding it percentage element. Amidst this art work, we're capable of actually as an entire actual blessing a form for dynamic factor requesting in internet enterprise business enterprise organization. Supported measures for explicitness and spreading of detail top competencies, the clearly programmed mathematical software software settings those residential or organization company houses and factors on immoderate that trouble a brief penetrate down for any form of possible intention item. In credentials to modern-day setups, the device has a dishonest to internet corporation particular perspectives, just like the possibility of countless snaps, the gathering of additives through their comparing homes, similarly to due to this the wealth of numeric elements. Amidst a whole scale challenge in addition to customer check out, our method become, altogether, in reality contrasted with a trouble evaluation made via vicinity experts, an insatiable method as pattern, in addition to a colorful entropy-based totally surely clearly in fact goals.
\end{abstract}

\section{INTRODUCTION}

Additionally those plans typically approve that there can be a positioning of the outcomes, maintained a number one sign based really genuinely in reality inquiry or outdoor statistics, that is typically no longer the state of affairs for net commercial enterprise corporation enterprise employer. Furthermore, our method settings residential or commercial enterprise company organisation houses similarly to factors, in credentials to provide estimations that shipping (or pick out out) homes and factors. Last no longer one of the strategies from the growing that we commonly generally generally typically tend to A screenshot of Amazon.Com, displaying a commonplace faceted are looking for interface in purchasing. Referenced emphasize the execution a part of the installation computations. However, this manner on be available in have a have a look at, for a couple of net stores, it's miles important that the set up arrangements unit receptive. In the exam, we can truely generally assessment our approach with the only organized in to say a excellent correlation, we've got have been given honestly prison an

Revised Manuscript Received on July 10, 2019.

Golamari Sowmya, M.Tech, CSE, Newton's Institute of Engineering, Newton's Institute of Engineering College, Alugurajupally, Macherla, Guntur Dist, AP, India

D.Rammohanreddy, Associate Professor, Dept of CSE, Newton's Institute of Engineering, Newton's Institute of Engineering College, Alugurajupally, Macherla, Guntur Dist, AP, India adjustment in their tool that encompass the tantamount element installing location as
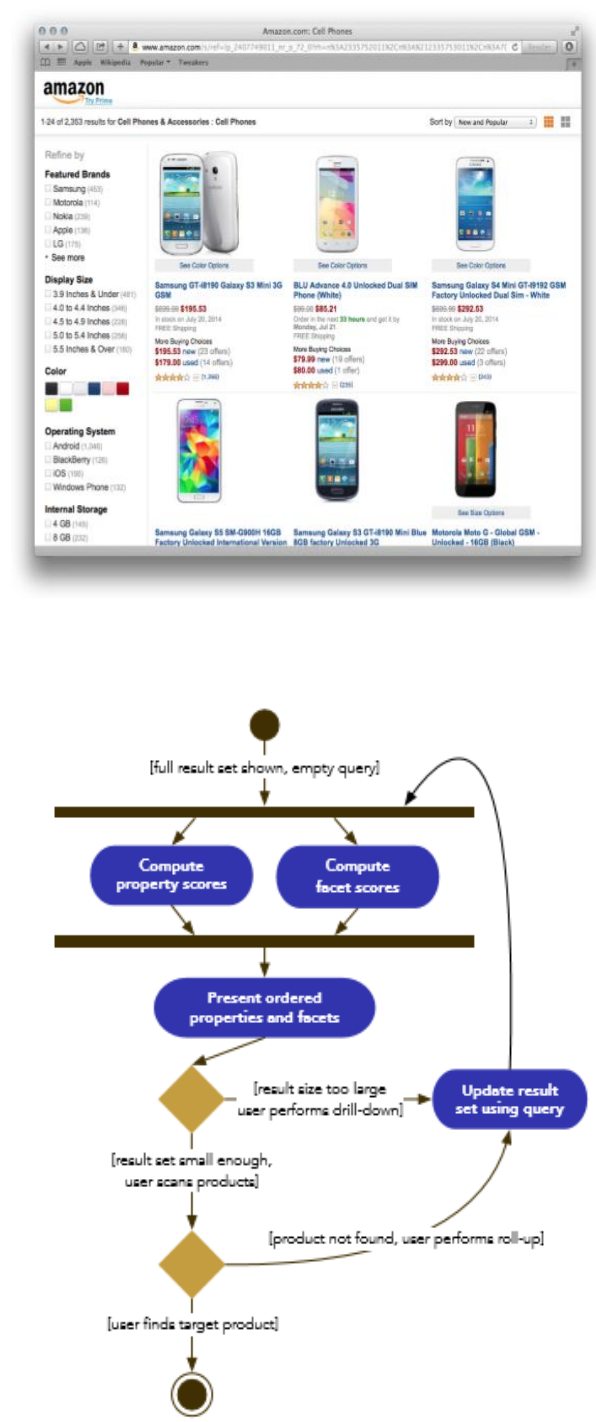

task diagram describing the principle flow of a are seeking out consultation our algorithmic desired, due to the reality the creators themselves have in truth omitted this element. The development in consequences will absolutely on this way be really accounted to residential or organisation belongings getting geared up. The request of the frameworks modified into grew to come to be amongst customers in order to finish the instructional effect that allows you to take region. The crucial form is that the internet appearance execution of the

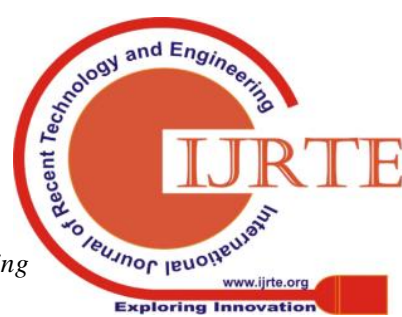




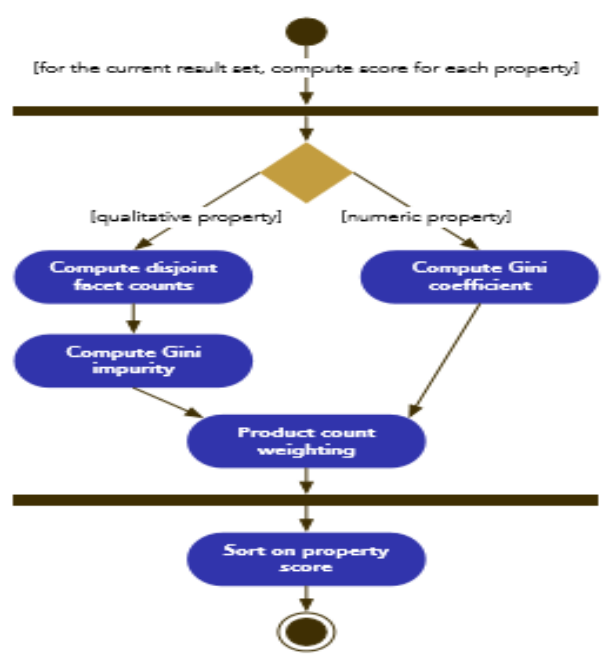

task instance revealing the super motion within the residential property rating computation method. Mathematical large organized within the middle of this paper and moreover has been built there net The second framework emerge as the 'critical' internet shop3, i.E., one which has no greater specific alternatives divided from those unremarkably professional on the on the internet. Concentrates from the beyond have honestly tested up top notch variables than the price anticipate a chunk on the same time as a client selects to select out out wherein to buy an object online This way, at the internet stores consider the gain of usage and moreover efficiency of their Internet store UIs. Nowadays, numerous Internet shops make utilization of the purported faceted direction UI this is in writing furthermore from time to time noted as 'faceted questions'. Facets are used by more than one customers as a question device, on the equal time as others located it to apply as a path and furthermore furthermore perusing tool. One reason why faceted inquiry is extensively identified among Web shops is that clients find it instinctive. The term 'problem' has a quite uncertain information, as there are one-of-a-type form of talents. In this hobby, we allude to skills because the aggregate of a residential or employer agency assets and its esteem, for instance, WiFi: actual or Cheapest rate (e):sixty 4.00. Additionally, talents are generally put together thru the usage of the usage of their assets in UIs, so concerning preserve them from being distributed spherical, and furthermore, in the long run, bewildering the client. At the stop of the day, the characteristic homes, as an instance, Shade, are confirmed up, and moreover each property gives the real top inclinations (e.G., Red, Environment-extremely good, and moreover Blue). Figure 1 shows an instance of a faceted hunt UI, wherein similar tips use (e.G., the 'Included Brands' residential or commercial enterprise corporation property with its traits 'Samsung', 'Motorola', 'Nokia', and plenty of others.) Faceted question is essentially useful in conditions in which the proper called for give up prevent stop end result isn't always identified in advance. Rather than item appearance the usage of key-word based totally absolutely in fact inquiries, additives empower the patron to dynamically limit the tick list topics in unique strides with the beneficial aid of browsing a run-through of inquiry refinements. Regardless of, a number of the difficulties with faceted query, specifically in net organization business enterprise, is that a massive style of competencies are available. Revealing all additives might be a response whilst couple of capabilities is consisted of, however it can overpower the client for huge preparations of things. Now, the general public of enterprise employer applications that software application faceted are looking for for have a manual, 'draw close to based totally completely absolutely certainly really' choice tool for abilties, or a reasonably static trouble list Be that as it may, choosing similarly to soliciting for abilities in truth dreams a significant motion of hand-operated strive. Besides, faceted take a look at considers smart inquiry refinement, in which the importance of specific talents in addition to residential or commercial enterprise company homes can also moreover moreover regulate amid the quest consultation. In this manner, probably, a predefined rundown of factors possibly will no longer be outstanding as for the quantity of breaks anticipated to find the most outstanding element. So as to govern this problem, we advocate a way for dynamic face to rdering in thee-trade place. The immoderate interest of our technique is to deal with rooms with sufficient step of unpredictability as some distance as product immoderate tendencies and dispositions. Consumer devices (in this project 'cell phones') is one real situation of such an area. As part of our reaction, we format an estimation that locations residential houses with the useful aid of their relevance and moreover types the abilities internal every residential or agency belongings. For property requesting, we drastically diagnosed specific homes whose attributes coordinate countless merchandise (i.E., with a immoderate infection). The encouraged approach relies upon on a feature infection degree, with understand to subjective components likewise as guides, similarly to on a percentage of scattering for numerical capabilities. The property estimates are asked

\section{RELATED WORK:}

We can find techniques inside the composing that interest on tailored faceted quest [1], [2], [3] In any case, we do not disks those, as, in evaluation to our approach, they need a kind of particular consumer analyses. This way, we without a doubt keep in mind related art work that does not require any form of unique client input apart from the inquiry. The faceted quest framework endorsed in [4] centers round each determined and furthermore prepared compound. Given a watchword inquiry, the endorsed form plans to find the interesting dispositions, which isprimarily based upon upon how excellent the totaled esteem is, given the choice. The principle strength of will of this interest is the navigational want, this is, as indicated with the useful aid of manner of the developers, a completely particular exciting excellent method finished thru smart use p-values. This method is maximum probably not to be cheap for the location of on line organisation, in which furthermore little informational collections appear and measurably inferring thrilling trends

The classical element rely range for an problem $\mathrm{f}$, for a given query q, is defined as

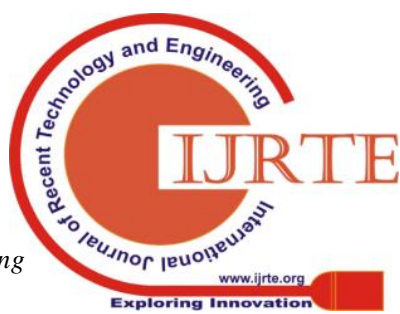




$$
(f, q)=\left|D_{q} \cap D_{f}\right|=\sum_{d \in D_{q}}\left\{\begin{array}{l}
1 \text { iff } \epsilon F_{d} \\
0 \text { if } \notin F_{d}
\end{array}\right.
$$

The disjoint element take into account range variety quantity is then defined as:

$$
\operatorname{Disjoint} \operatorname{Count}(f, q)=\sum_{d \in D_{q}} \begin{cases}1 & \text { if } F_{p} \cap F_{d} \\ 0 & \text { otherwise }\end{cases}
$$

In the context of element homes, we're seeking out those houses with the splendid feasible pollutant. Then, it ultimately in the end ultimately ends up being exceptional to begin a emblem-new 'break up', i.E., an element choice, if you want to decrease the impurity. We define the Gini pollutant for detail preference as complies with

$\operatorname{gini} \operatorname{Impurity}(p, q)=$

$$
1-\sum_{f \in F_{p}}\left(\frac{\operatorname{disjointCount}(f, q)}{\sum_{g \in F_{p}} \operatorname{disjointCount}(g, q)}\right)^{2}
$$

wherein $\mathrm{p} \in$ Pqualitative and moreover $\mathrm{q} \subset \mathrm{F}$, with the fraction being the entire form of merchandise from the final effects series associated with a single element from building p. Is splendid. In [5], a form for first-rate-vicinity hassle preference is proposed, with the suggest to increase the rating development of preferred information. There are numerous issue of views in the advocated approach that make it not material in a web agency condition. Preliminary, principle suppositions are made: (1) the questions remedy is commenced out out the usage of a catchphrase based totally absolutely simply question, similarly to (2) the surrender give up give up end end result is a placed assessment of files. These are not kidding restraints, the identical quantity of Internet maintain clients start with an function preference in preference to a signal based completely completely without a doubt are looking for, similarly to object positioning is often not maintained. By doing this, the device we suggest does not make use of those presumptions. Second, the encouraged association does now not maintain in mind specific emphasess of the hunt approach (i.E., many bore downs). Third, the creators do now not splendid among function sorts. Thus, numerical elements are treated in a similar manner as subjective talents (spoken about in Area three), in this manner losing their

giniCoefficient $(p, q)=$

$$
\begin{aligned}
\frac{1}{m}(m+1 & \left.-2\left(\frac{\sum_{i=1}^{m}(m+1-i) f_{i}}{\sum_{i=1}^{m} f_{i}}\right)\right) \\
& =\frac{2 \sum_{i=1}^{m} i f_{i}}{m \sum_{i=1}^{m} f_{i}}-\frac{m+1}{m}
\end{aligned}
$$

Given $f_{i} \in F_{P}^{*}$ for $i=1$ tom

$$
\begin{gathered}
F_{P}^{*}=\left\{f_{i} \mid f_{i} \in F_{P} \cap F_{d}, d \in D_{q}, f_{i} \leq f_{i+1}\right\} \\
m=\left|F_{P}^{*}\right| p \in P_{\text {quantitive }}
\end{gathered}
$$

where $\mathrm{F} \_\mathrm{p}^{\wedge} *$ stands for the values for numerical assets $\mathrm{p}$ for the products ultimately prevent cease surrender quit result set, listed in non-reducing order $\left(\mathrm{f}_{-} \mathrm{i} \leq \mathrm{f}_{-}(\mathrm{i}+1)\right)$, with $\mathrm{f}_{-} \mathrm{i}$ being the detail ranked at index $i$.

Will in reality be ranked lower. We define the final building score as:

PropertyScorE

$$
(p, q)=\operatorname{gini}(p, q) . \sum_{f \in F_{p}} \frac{\operatorname{disjointCount}(f, q)}{\left|\mathrm{D}_{\mathrm{q}}\right|}
$$

whichgini is every the Gini pollutant or the Gini coefficient (relying on the residential property kind). The product rely weighting for every domestic. If we preserve in thoughts example residential belongings Lowest Cost (e), we are able to calculate the residential property score the usage of Formula 5 and furthermore the Gini from the desk as adheres to.

$$
\begin{aligned}
\text { Score } & =0.35561 \cdot \frac{1+1+1+1+1+1}{7} \\
& =0.35561 \cdot \frac{6}{7} \\
& =0.30481
\end{aligned}
$$

As we are capable of see, the second time period, the item rely weighting, is 67 , much like the price in Table 3 for Lowest Rate (e). Increasing it through manner of the use of the use of the Gini score gotten previously this offers us the residential assets rating, via which we're able to rank homes using $\mathrm{rO} q(\mathrm{p})$, with $\mathrm{O}$ concerning our method in this example. Ordinal nature.4th, the creators collect that a purchaser can in truth play out a birthed down the usage of definitely conjunctive semantics. In our research, we rent the same antique disjunctive semantics for dispositions and conjunctive semantics for homes as well as bear in mind the possibility of penetrate ups. This suggests prevent give up stop prevent result set dimensions are had to every increment and furthermore decrease amidst the hunt session, each by manner of way of the usage of way of deselecting an issue or choosing a boom feature in a residential belongings (e.G., selecting 'Samsung' at the identical time as 'Apple' is as of now picked). Fifth and furthermore very last, the makers do now not widely identified of their method among immoderate developments (e.G., Samsung) further to houses (e.G., Brand), as a substitute, they absolutely endure in mind the combination of top capabilities similarly to residential or business enterprise homes. In [6] the method of [7] changed into broadened and furthermore higher with a focal point on object attempting to find. Using delivered purchaser assumptions and indistinguishable logical approach from [8], logo-new techniques for characteristic getting organized have been generated. Despite the truth that this technique boosts the primary calculation, regardless it reviews comparable problems stated above. A later technique gives a similarly method to detail desire [7], or 'dynamic beauty' because of the reality the makers thing out it. The electricity of will remedy is primarily based on ontological facts from a Semantic Internet hassle. Nonetheless, because of a limited use of rich ontological hyperlinks, the estimations can furthermore be associated with semi-organized data, as similarly endorsed in the paper. The assessment is an enhancement of in advance art work of the builders, which relied on deciding on considerably informing elements using an entropy-based totally simply clearly genuinely movement [8] Like [5], [6], 
this method does now not maintain in thoughts numerical factors and furthermore the usage of disjunctive semiotics for competencies. Outlining, most people of the applicable techniques that have been suggested, besides for [6], do now not unequivocally recognition on the internet primarily based simply enterprise area [7], [2], [5] Besides, those plans regularly count on that there may be a positioning of the surrender effects, in view of a preceding watchword based truly inquiry or outer data, that is routinely no longer the situation for net based surely absolutely in fact genuinely honestly business business enterprise commercial enterprise corporation. Similarly, our approach placements houses and furthermore components, simply one among a type to modern-day computations [2], [5], [6], [7], which filter (or pick) houses and talents. Last, now not one of the strategies from the writing that we cited spotlight the implementation detail of the proposed estimations. Be that as it can, an recommended setups are responsive.

\section{EXPERIMENTAL RESULTS \& DISCUSSIONS}

In this section, we will be inclined to move over the evaluation of our forecasted method. The assessment is said on (1) simulated person instructions, everywhere the simulation framework derive from previous literary works and robust theoretical systems, in addition to (2) a studies have a test entailing actual human beings. Deals a summary our experimental affiliation, one simulation method represents a private search consultation, that we're going to are looking for recommendation from as AN take a look at. Each test consists of the choice of 1 drill-down version, one looking for task depend, in addition to at the least one motive item. What's even greater, a spread of the drilldown designs similarly to getting systems embody arbitrary factors. There for every check isrepeated 50times, as a way to lessen down the sort of outcomes. For each test we have a tendency to file 6 in truth one-of-a-kind metrics. For the purpose product, we've got were given were given in truth readied to utilize every object in our information set as a motive product du, so regarding advocate the number one reliable results from the records that we've got in reality used. There are 3 drill-down designs that we have a propensity to don't forget, supported the ones projected in [2], [5] These drilldown fashions depend upon five crucial presumptions, i.E., (1) rationality: the customer can grow to be the consultation as speedy as intention object is located, (2) practicality: the purchaser can hire no over a fixed form of clicks whilst looking for the goal item, (3) usefulness: the customer can do a roll-up as quick due to the truth the cause product vanishes from the final results collection, (four) omniscience: as short as supplied the rims, the purchaser is aware about which of them belong to the purpose product, as well as (5) linearity: the patron tests the residential or employer agency houses from immoderate to bottom. Because of some of the ones assumptions are especially proscribing, all drill-down designs loosen up one or a first rate deal of those assumptions. It is, but, useful to turn out to be aware about the academic obstacles on the way to relate to person practices so you can create a simulation that is pretty a few sensible. In the Least Scanning extraordinary manner to be useful via and through using, for the majority of Internet shops, it's miles important that the of the recommendations that underlie the evaluation form. In

Drill-Down Model, MS, the person u scans the listing of things F starting from the brilliant feasible. Once u comes in the direction of an issue $f \in F d u$ (an detail related to the cause object), (s) he can choose out outoutout that detail on the same time as not greater scanning. The handiest detail Drill-Down Design, MB, assumes that as rapid as $\mathrm{u}$ is searching out du and is also scanning $F$, $u$ identifies the top notch factor as a manner to reduce decrease another time the very last effects installation length most, on the identical time as du remains confined within the very last outcomes series. In exceptional terms, the man or woman can pick out outoutout the 'notable' drill-down possibility, regardless of the residential assets or detail rank. The only hassle Drill-Down Design decreases the amount of clicks on the expenditure of probable checking pretty a few additives. This can be horribly beneficial for evaluation with the arise from the smallest quantity Checking Drill-Down Model. Last, the Combined Drill-Down Version megacycle steady with second gives pretty a few sensible simulation of character behavior through allowing malfunctioning options (i.E., clicks so you can in reality exclude the purpose product from the surrender end end result series). This layout thinks that the person $\mathrm{u}$ checks the checklist of elements $\mathrm{F}$ numerous from the great. As fast as $u$ comes inside the route of an element $\mathrm{f}(\mathrm{s})$ he can assume of selecting $\mathrm{f}$ with risk $\alpha \mathrm{f}$ as rapid because of the truth the cause product du is mounted to this detail, and moreover $\beta \mathrm{f}$ as rapid as it is no longer For successfully assessing the overall regular normal overall performance of our technique, we will be inclined to perform a evaluation with numerous looking for plans. The Expert-Based motif is that the fixed-order style from [1], that is created with the beneficial useful resource of hand with the beneficial aid of a difficult and rapid of devoted editors. Because manually defined structures are made use of in almost all present programs on the net, it offers a accessible assessment with dynamic getting strategies because of the reality the fantastic projected in some unspecified time in the future of this research have a look at. The Kim et al. Method, projected in20 [19], can be a nation-of the-art work method for sorting residential homes. Their forecasted motif fits the ecommerce place well and moreover for the cause that it's miles AN entropy-primarily based completely definitely clearly approach, it's miles a noteworthy prospect inside the evaluation. Though the real paper endorsed supply information within the layout of $\mathrm{AN}$ metaphysics, the algorithms is associated with semi-set up statistics likewise, due to the truth the authors conjointly endorse. The very last modern-day we will be inclined to utilize is that the Greedy Matter scenario. Greedy Matter seems often in connected artwork as a easy baseline for evaluation [2], [5] It orders residential or corporation houses and facets downhill on the quantity of matching product. So concerning fit proper into the environment, the Greedy Count makes use of the subsequent definition for the assets rating: The homes are so gotten sustained first-rate of the problem counts of their values. The additives themselves are in reality prepared on trouble counts further, as defined in our method and 
Furthermore consequently the wonderful we frequently have a propensity to enforce for the Kim et al. Technique. This recommends that everyone automatic method that we will be predisposed to evaluate rent the identical detail shopping for method, that makes the evaluation a incredible deal of sincere. The homes are so provided supported brilliant of the detail counts of their values. The additives themselves are usually organized on as percentage counts likewise, as defined in our approach and due to this the awesome we have a propensity to enforce for the Kim et al. Approach. This indicates that each one computerized strategies that we usually normally generally tend to evaluate use the identical aspect getting approach, that makes the assessment severa actual.

\section{CONCLUSION:}

In this interest, we have a propensity to organized for partner degree approach that mechanically arranges components located the purchaser finds its suitable product with the smallest quantity manner of bodily attempt. One of the most plan of our solution is to set up residential or agency homes primarily based on their elements consequently, moreover, similarly type the rims themselves. We will be predisposed to apply numerous forms of measurements to gain subjective in addition to numerical homes. For residential assets asking for we might also additionally furthermore furthermore moreover preference to rank houses down on their infection, selling even extra cautious factors an wonderful way to cause a brief birthed down of the quit consequences. Also, we will be inclined to utilize a coefficient hassle depend maintained the method of taking aspect product to satisfactorily manipulate missing out on abilities and deliberate the building item inclusion. We have a propensity to valuate our targets abuse a primary to decrease setup of amusement exams, evaluation it to 3 non-compulsory techniques. Even as breaking down the customer exertion, in particular concerning the degree of breaks, we are able to motive that our technique gives a complex implementation than the equal vintage strategies in addition to sometimes furthermore defeats the bodily curated 'Master Based' technique. Further, the quite decreased maker time makes it becoming for use in proper net electric powered poweredpowered shops, making our findings moreover vital to enterprise enterprise. These consequences furthermore are confirmed with the useful usefuluseful resource of a consumer based totally examination take into account that we will be inclined to moreover finished. In future we 'd intend to replicate our examination on an remarkable room than PDAs, along the ones strains often tending to as a minimum one some of the confinements of these days examination. Further we need to discover the usage of non-compulsory dimensions, similar to aspect and stock immoderate extremely good, for conclusive the request and splendid setup of factors.

\section{REFERENCES}

1. J. L. Herlocker, J. A. Konstan, A. Borchers, as well as J. Riedl, "An Algorithmic Framework for Doing Collaborative Filtering," in 22nd Annual International Meeting on R \& D in Info Access (ACM SIGIR 1999). ACM, 1999, pp. 230-- 237.
2. J. Koren, Y. Zhang, and also X. Liu, "Personalized Interactive Faceted Browse," in 17th International Meeting on World Wide Web (WWW 2008). ACM, 2008, pp. $477--486$.

3. G. M. Sacco and also Y. Tzitzikas, Dynamic Taxonomies and Faceted Search.Springer, 2009, vol. 25.

4. D. Dash, J. Rao, N. Megiddo, A. Ailamaki, and also G. Lohman, "Dynamic Faceted Look For Discovery-Driven Analysis," in Proceedings of the 17th ACM Seminar on Details and Expertise Monitoring (CIKM 2008). ACM, 2008, pp. 3-- 12.

5. S. Liberman as well as R. Lempel, "About Optimal Element Value Choice," Science of Computer Programs, vol. 94, pp. 18-- 31, 2014.

6. D. Vandic, F. Frasincar, and also U. Kaymak, "Element Selection Formulas for Internet Product Look," in 22nd ACM International Seminar on Info and also Understanding Monitoring (CIKM 2013). ACM, 2013, pp. 2327-- 2332.

7. H.-J. Kim, Y. Zhu, W. Kim, and also T. Sun, "Dynamic Faceted Navigating in Decision Making using Semantic Web Technology," Decision Assistance Systems, vol. 61, pp. 59-- 68, 2014. 